\title{
Nuevos retos en la Enfermería Cardiovascular
}

\author{
New challenges in Cardiovascular Nursing
}

Según la Organización Mundial de la Salud (OMS), la patología cardiovascular es la principal causa de muerte a nivel mundial, su prevalencia, coste económico y social sigue en aumento a pesar que los factores causales son conocidos (1). El envejecimiento de la población favorece el aumento de pacientes que presentan un acúmulo de este tipo de enfermedades; tal es así que, se prevé que en el 2030, las patologías cardiovasculares sigan siendo la principal causa de muerte en el mundo.

En los últimos años, la investigación científica está logrando importantes avances en el tratamiento de las enfermedades del corazón y grandes vasos, y aun cuando se están usando cada vez más equipos sofisticados para el soporte y/o asistencia a pacientes cardiacos cuyo estado es muy comprometido, la tendencia es ser cada vez menos invasivos $(2,3)$. Es así que hoy en día las salas de hemodinámica y en menor proporción las unidades de cuidados intensivos coronarios y cuidados intensivos generales, son las áreas donde se realizan la gran mayoría de procedimientos a los pacientes con problemas cardiovasculares, quedando el uso de quirófanos reservados solo para contadas cirugías cardiacas. En todas estas áreas siempre está presente la enfermera como responsable del proceso del cuidado del paciente durante la ejecución de los procedimientos ymientras dure su hospitalización.

Los avances más importantes de la cardiología, se han dado en el campo de la enfermedad coronaria, debido a que es la patología cardiovascular con mayor prevalencia,se han desarrollado técnicas para eliminar obstrucciones de las arterias coronarias, tales como: los balones de angioplastia y los stents conteniendo fármacos para evitar una nueva oclusión; y están en estudio, el stent biodegradable, el cual cumplida su función se eliminaría de la arteria sin dejar rastro.En relación a la muerte súbita por arritmias malignas, están los desfibriladores automáticos implantables y para pacientes con fallo de la función de bomba del corazón, está el re-sincronizador cardiaco, que estimula de manera sincronizada diferentes partes de la pared ventricular logrando una contracción más eficaz. Todos estos procedimientos se realizan en la sala laboratorio de hemodinámica y luego se completa el cuidado en la unidad coronaria.

Para la insuficiencia cardiaca, están las drogas inotrópicas orales y para las fallas más complicadas, están los dispositivos de asistencia ventricular izquierda implantables, que son bombas mecánicas que realizan la función de bombeo, cuando los medicamentos y marcapaso ya no ayudan al corazón (4).Y lo más reciente, la Oxigenación por Membrana Extracorpórea (ECMO) que es una técnica de asistencia circulatoria, una terapia de rescate altamente invasiva, destinada a pacientes críticamente enfermos, con insuficiencia respiratoria grave y fallo cardiovascular asociado o sin él (5). Estos pacientes son cuidados en las unidades de cuidados intensivos coronarios o en la UCI general. Esto genera la necesidad que la Enfermera especialista en cardiología, tenga los conocimientos y habilidades necesarias para brindar un cuidado holístico.

Por otro lado para el diagnóstico y evaluación de la función del corazón, la ecocardiografía tridimensional, técnica que permite la visualización de la imagen cardiaca en tiempo real, es quizás uno de los avances más importantes en el apoyo del diagnóstico de las enfermedades cardiacas, pues permite una visión detallada del corazón, los grandes vasos y de la actividad eléctrica. Esta es otra área para la enfermera especialista en cardiología, en la sala de estudios con medios no invasivos. 
Sabido es que a pesar de toda la tecnología y los avances terapéuticos, es la vida sana promovida por la medicina preventiva la que va a abaratar los costos y mejorar las calidad de vida de la persona con patología cardiaca, y en este aspecto hay que resaltar el rol protagónico de la enfermera en el Programa de rehabilitación cardiaca y en el Consultorio de insuficiencia cardiaca, haciendo prevención primaria y secundaria, donde educa y orienta al paciente y familia para mejorar la calidad de vida.

Mg. Patricia Soledad Obando Castro ${ }^{1, \mathrm{a}}$

\section{REFERENCIAS BIBLIOGRAFICAS}

1. World Health Organization. Global status report on non communicable disaeses 2010. Geneva: World Health Organization. 2011.

2. Kérouac S. El pensamiento enfermero. 1 ${ }^{\text {a }}$. Edición. Madrid, España: Ed. Masson. 2001.

3. Cunha A. Enfermagem na Cardiologia Invasiva. São Paulo: Ed. Atheneu. 2007.

4. Paredes AM, Suarez A, Sánchez N, Martínez G. Dispositivos de asistencia circulatoria mecánica, estado actual. Revista Cubana de Cardiologia y Cirurgia Cardiovascular. 2010; 16(4):452-65.

5. Domínguez D, Salas G, Valdez M, et al. Oxigenación por membrana extracorpórea (ECMO): Experiencia en una unidad de cuidado intensivo neonatal. Arch. Argent. Pediatr. 2012; 110 (5):404410. 